\title{
DIVERSIDAD DE ORQUÍDEAS DE LAS DIFERENTES FORMACIONES VEGETALES DE LOS ANDES PERUANOS
}

\author{
Delsy Trujillo \\ Investigadora asociada, Herbario MOL, Facultad de Ciencias Forestales, Universidad Nacional Agraria La Molina. \\ Av. La Universidad s/n. La Molina. Apartado 12-056 - Lima, Perú \\ delsytrujillo@lamolina.edu.pe
}

\begin{abstract}
Resumen. Debido a la presencia de la Cordillera de los Andes y la Corriente de Humboldt, el Perú posee una gran diversidad de ecosistemas. Mientras que las regiones bajas al oeste de los Andes peruanos está caracterizada por ser una franja desértica-semidesértica, con bosques secos, matorrales y principalmente formaciones vegetales estacionales, las regiones del lado este de los Andes se caracterizan por estar formados de extensos bosques húmedos de gran biodiversidad. A lo largo de la cordillera; la vegetación de la zonas altoandinas y de los valles interandinos del norte, centro y sur del Perú no son tan contrastantes, aunque si existen diferencias en la composición florística debido a la humedad; las regiones del norte son mas húmedas que las del centro y sur. Las orquídeas que se encuentran en los bosques montanos de la vertiente oriental de los Andes peruanos son principalmente epífitas y han sido objeto de numerosos estudios con el propósito de conocer su diversidad. En cambio el estudio de las orquídeas terrestres no han recibido la misma atención, a pesar de que éstas se encuentra en casi todos los ecosistemas del país, desde los desiertos, matorrales xerofíticos, turberas altoandinas e incluso en áreas donde se ha introducido especies exóticas, como los bosques de Eucalyptus globulus. En la presente contribución se describen las formaciones vegetales del desierto costero, valles interandinos y zonas altoandinas del Perú; así como las orquídeas que se han registrado en cada tipo de vegetación.
\end{abstract}

Aвstract. Due to the presence of the Andes Cordillera and the Humboldt Current, Peru has a great diversity of plant communities. For example, while the lowlands to the west of the Peruvian Andes are characterized mainly by being a semi-desert belt with dry forest, shrublands, and mostly seasonal vegetation formations, the eastern side of the Andes is covered by a vast wet forest of high biodiversity. In contrast to the lowlands on either side of the Andes, the Andean highlands throughout Peru are not as contrasting, although there are floristic differences because of diminishing humidity toward the south. Epiphytic orchids, which are distributed mainly in the montane forests of the eastern slopes of the Peruvian Andes, have been the goal of numerous botanical expeditions in order to document their diversity. By contrast, Peruvian terrestrial orchids have not received the same attention, although they are found throughout the country, from the deserts, xerophytic shrublands, wetlands at high elevations, even in areas where exotic species such as Eucalyptus globulus forest have been introduced. The present contribution describes the vegetation of the desert coast, inter-Andean valleys and highlands of Peru, as well as orchids that have been recorded in each vegetation type.

PALABRAS ClaVE: Orchidaceae, Perú, desierto costero, zonas altoandinas, valles interandinos

La Cordillera de los Andes y la Corriente de Humboldt hacen que el Perú albergue una gran diversidad biológica. La primera crea una variedad de zonas ecológicas entre sus estribaciones y la segunda modifica notablemente las condiciones térmicas y precipitaciones pluviales al lado oeste de los Andes, ocasionando la aridez de la costa peruana (Weberbauer 1945; Dillon et al. 2003).

Una característica de los Andes peruanos es la notoria diferencia que existe en la vegetación a ambos lados de la cordillera. Mientras que las formaciones herbáceas estacionales, matorrales y bosques xerofíticos se desarrollan en la vertiente occidental, los bosques húmedos tropicales caracterizan la vertiente oriental.

La mayor diversidad de orquídeas se encuentran en los bosques de las vertientes orientales; éstas son principalmente epífitas (muchas con flores llamativas) y han sido por décadas el interés y objeto de investigación de muchos botánicos y aficionados 
al cultivo de las orquídeas. Por tal razón existe más información de las orquídeas de esta región de los Andes peruanos, que de las vertientes occidentales, zonas altoadinas y valles interandinos. Con el propósito de incrementar el conocimiento de la diversidad, distribución y estado de conservación de las orquídeas terrestres de la costa y territorio Andino (sierra) del Perú; en los últimos años se ha ido procesando información a partir de trabajos de campo, revisión de herbarios (USM, MOL, HAO, HUT, CPUN y PRG) y datos proporcionados por diferentes botánicos peruanos. Así como consultas a las bases de datos online de F y MO en busca de registros adicionales. Producto de los datos procesados, a continuación se describe las diferentes formaciones vegetales de la costa, zonas altoandinas y valles interandinos; así como las orquídeas que se han reportado en cada una de ellas.

\section{Vegetación de la costa}

La costa peruana es una angosta faja desérticasemidesértica que se extiende desde $3^{\circ} 23^{\prime}$ hasta los $18^{\circ}$ latitud sur y ocupa aproximadamente el $10 \%$ del territorio peruano. La altura sobre el nivel del mar es variable, pero no sobrepasa los $1000 \mathrm{~m}$ de altitud (Ferreyra 1983). La condición desértica-semidesértica en la costa está en íntima relación con la influencia de la Corriente fría de Humboldt y el efecto de sombra de la Cordillera de los Andes (Dillon et al. 2003).

La vegetación semidesértica de la zona costera comprendida entre los $4^{\circ}$ y $8^{\circ} \mathrm{S}$ está influenciada por la ocurrencia de lluvias durante los meses de diciembre a marzo (verano); las cuales pueden ser muy abundantes durante la ocurrencia de los eventos El Niño y causar grandes inundaciones. En esta zona se observa un marcado estrato arbóreo y arbustivo xerofítico que puede o no perder las hojas durante la estación seca y un estrato herbáceo efímero, que solo aparece durante la época de lluvias. De acuerdo a la literatura y material de herbario que se ha revisado, en éstos bosques estacionalmente secos, hasta la fecha no se ha registrado especies de orquídeas. Esto quizá se debe a que las investigaciones en estas formaciones vegetales se han enfocado al estudio de las especies leñosas (Linares-Palomino 2004; Aguirre et al. 2006; La Torre-Cuadros \& Linares-Palomino 2008).

A partir del grado $8^{\circ} \mathrm{S}$, la costa peruana es una región hiperárida más o menos continua que ocasionalmente es interrumpida por los ríos que descienden de la Cordillera de los Andes. En esta zona de los desiertos, a pesar de que la humedad atmosférica es alta todo el año, las precipitaciones son escasas y solo se presentan a manera de una lluvia fina interrumpida; por lo que la vegetación que se desarrolla es fundamentalmente de tipo herbácea. Las formaciones vegetales donde se han registrado orquídeas son:

Formaciones de Lomas - Son comunidades de plantas efímeras que se desarrollan en ciertas colinas cercanas al mar y que rara vez sobrepasan los $1000 \mathrm{~m}$. de altitud. La vegetación se desarrolla entre los meses junio y octubre (invierno y parte de la primavera) debido a la condensación del manto de neblina que cubre el litoral peruano por efecto de la corriente de Humboldt (Ferreyra 1983; Dillon et al. 2003). Aunque por lo general en el Perú las lomas se encuentran distribuidas discontinuamente entre los $8^{\circ}$ y $18^{\circ} \mathrm{S}$ (Ferreyra 1983, 1993), éste tipo de formación vegetal también se ha registrado más al norte, a los $6^{\circ} 52^{\prime} \mathrm{S}$ en el Cerro Reque (Weberbauer 1945; Dillon et al. 2003).

La vegetación de lomas está compuesta principalmente por especies herbáceas anuales y perennes (geófitas); aunque existen localidades con especies leñosas. La composición florística varía de una localidad a otra, así como su grado de desarrollo. En las lomas del norte llegan a su óptimo desarrollo en julio, mientras que las del sur en octubre-noviembre; dependiendo esto de la cantidad de precipitaciones invernales, la cual varía de un año a otro.

Con respecto a las orquídeas que se han registrado para las formaciones de lomas (Tabla 1), Chloraea pavonii es la más conocida y vistosa; ésta orquídea era frecuente en las lomas cercanas a la ciudad de Lima (capital del Perú), por lo que no pasaba desapercibida y fue colectada por los principales botánicos y naturalistas que han estudiado la flora peruana como H. Ruiz \& J. Pavón, A. Raimondi, A. Weberbauer y R. Ferreyra (Ruiz 1952; Pupulin 2012). En la actualidad esta orquídea parece haber desaparecido de las formaciones de lomas cercanas a la ciudad de Lima debido a la expansión urbana, siendo el ultimo espécimen que se ha examinado en los herbarios el de Ferreyra 8748 (USM) de 1952 en Lomas de Iguanil. 
TABLA 1. Localidades de lomas donde se han registrado especies de orquídeas. Las localidades han sido ordenadas de norte a sur.

\begin{tabular}{|c|c|c|}
\hline Localidad & Especies & Voucher / Referencia \\
\hline \multicolumn{3}{|c|}{ Departamento de Lambayeque } \\
\hline Cerro Reque & Pterichis sp. & $\begin{array}{l}\text { Laos } 2051(\mathrm{USM}) \\
\text { Laos } 4574(\mathrm{PRG}) \\
\text { Laos } 4603(\mathrm{PRG}) \\
\text { Llatas } 342(\mathrm{MO})\end{array}$ \\
\hline \multicolumn{3}{|l|}{ Departamento de La Libertad } \\
\hline Cerro Campana & Pelexia matucanensis (Kraenzl.) Schltr. & $\begin{array}{l}\text { Angulo } 765 \text { (HUT) } \\
\text { López } 710 \text { (HUT, F) }\end{array}$ \\
\hline \multirow[t]{2}{*}{ Cerro Cabras } & Malaxis andicola (Ridl.) Kuntze & Angulo s.n. (HUT) \\
\hline & Pelexia matucanensis (Kraenzl.) Schltr. & Angulo 1309 (HUT) \\
\hline \multicolumn{3}{|l|}{ Departamento de Ancash } \\
\hline Lomas de Casma & Pelexia matucanensis (Kraenzl.) Schltr. & Ferreyra 8049 (USM, MOL, F) \\
\hline Lomas de Mongon & Pelexia matucanensis (Kraenzl.) Schltr. & Leiva et al. 2008 \\
\hline \multicolumn{3}{|l|}{ Departamento de Lima } \\
\hline Lomas de Lachay & Aa weddelliana (Rchb.f.) Schltr. & $\begin{array}{l}\text { Cano } 7101 \text { (USM) } \\
\text { WENT s.n. (MO) }\end{array}$ \\
\hline Lomas de Iguanil (Chancay) & Chloraea pavonii Lindl. & Ferreyra 8748 (USM, F) \\
\hline Lima $^{a}$ & Chloraea pavonii Lindl. & $\begin{array}{l}\text { Raimondi } 471 \text { (W) } \\
\text { Ruiz s.n. (G) }\end{array}$ \\
\hline \multirow[t]{2}{*}{ Amancaes } & Pelexia matucanensis (Kraenzl.) Schltr. & Esposto s.n. (MOL) \\
\hline & Chloraea pavonii Lindl. & $\begin{array}{l}\text { Esposto s.n. (MOL) } \\
\text { Weberbauer } 746 \text { (F foto) }\end{array}$ \\
\hline San Jeronimo & Chloraea pavonii Lindl. & Maisch s.n. (USM) \\
\hline Cerro Agustino & Pelexia matucanensis (Kraenzl.) Schltr. & $\begin{array}{l}\text { Soukup } 2045 \text { (USM, F) } \\
\text { Weberbauer } 5696 \text { (F) }\end{array}$ \\
\hline Lomas de Atocongo & Pelexia matucanensis (Kraenzl.) Schltr. & Ferreyra 9514 (USM, F) \\
\hline \multicolumn{3}{|l|}{ Departamento de Ica } \\
\hline Lomas de Amara & Aa weddelliana (Rchb.f.) Schltr. & Orellana 353 (MOL) \\
\hline Al sur de Nascaa & Aa weddelliana (Rchb.f.) Schltr. & Rahn 198 (USM) \\
\hline \multicolumn{3}{|l|}{ Departamento de Arequipa } \\
\hline Lomas de Los Cerrillos & Aa weddelliana (Rchb.f.) Schltr. & Ferreyra 13455 (USM) \\
\hline Atiquipa & Aa weddelliana (Rchb.f.) Schltr. & $\begin{array}{l}\text { Delgado } 4021 \\
\text { Ferreyra } 14034 \text { (USM) }\end{array}$ \\
\hline
\end{tabular}

Las demás orquídeas que se han reportado para lomas poseen flores menos conspicuas y por ende poco conocidas, estas son: Aa weddelliana (Fig. 1A), Malaxis andicola y Pelexia matucanensis (Schweinfurth 1958; Leiva et al. 2008; Trujillo \& Delgado 2011).
La revisión de las colecciones de los herbarios USM y PRG evidencia la presencia de poblaciones de Pterichis sp. creciendo de 400 a $585 \mathrm{~m}$ de altitud entre Tillandsia sp. en las lomas del Cerro Reque (Departamento de Lambayeque). Este registro, no 

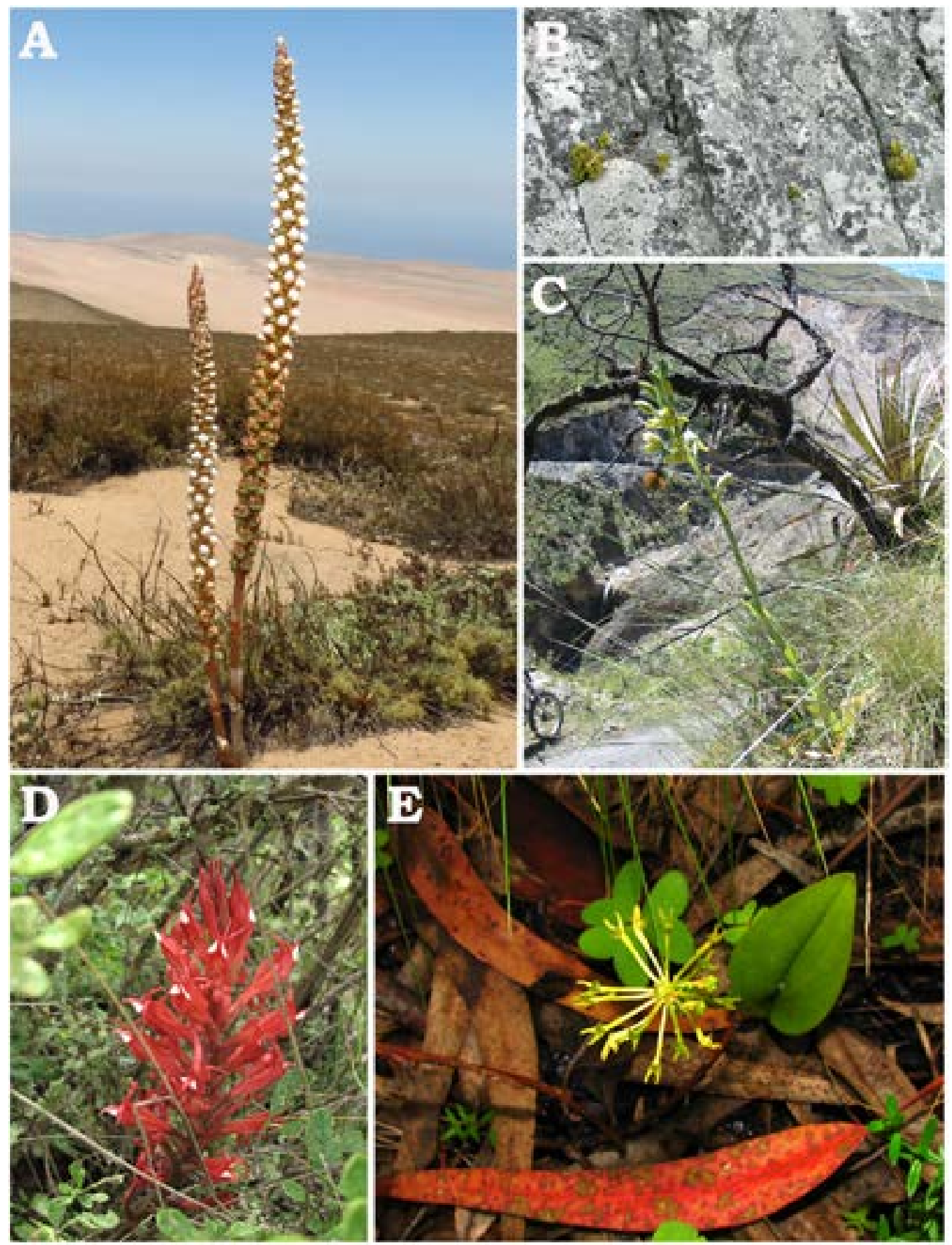

Figura 1. Fotografías de: A. Aa weddelliana en formación de lomas. B. Plantas de Stelis sp. creciendo sobre roca a $4000 \mathrm{~m}$ en la sierra de La Liberad. C. Chloraea septentrionalis creciendo al borde del camino. D. Porphyrostachys pilifera creciendo bajo arbustos. E. Malaxis andicola creciendo en el sotobosque de una plantación de Eucalyptus globulus. Fotos: D. Trujillo. 
sólo representa una nueva especie de orquídea para las formaciones de lomas del Perú, sino también permite ampliar el rango de distribución altitudinal del género Pterichis, que se pensaba que sólo se encontraban entre los 2800 a $4300 \mathrm{~m}$ de altitud (Cribb 2003).

Tillandsiales - Son comunidades vegetales muy especiales, constituidas por una o varias especies del género Tillandsia (Bromeliaceae) que habitan sobre las arenas de los desiertos entre $8^{\circ}-18^{\circ} \mathrm{S}$. Estas plantas tienen la particularidad de absorber el agua no por las raíces, que están atrofiadas, sino por las hojas a través de unos pelos escamosos; por lo que enteramente dependen de la humedad del ambiente (Ferreyra 1983; Hesse 2012). Los Tillandsiales se encuentran en las colinas de suave pendiente, adyacentes al territorio de las lomas costeras a manera de una angosta faja gris discontinua, comúnmente ocupa áreas muy reducidas, aunque hay algunas que son de gran extensión. En ésta formación vegetal, la única orquídea que se ha registrado es una $A a$ sp. en los Tillandsiales de Marcona, Departamento de Ica (Vector-Peru 2009; C. Vargas, comm. pers.); es muy probable que la identidad de ésta especie sea $A$. weddelliana, que es la especie que se ha registrado en las formaciones de lomas del mismo departamento.

\section{Vegetación del territorio andino}

El territorio andino o sierra se extiende desde los 800 o $1000 \mathrm{~m}$ de altitud hasta el límite inferior de las nieves perpetuas $(\sim 5000 \mathrm{~m})$. La diversidad florística de los Andes peruanos es el resultado de su compleja orografía y de la variedad de climas que existe a lo largo de la cordillera (Weberbauer 1945). Mientras que la abundancia de precipitaciones caracterizan a las vertientes orientales y se presentan prácticamente durante todo el año; en las vertientes occidentales (parte superior de ellas), las zonas altoandinas y los valles interandinos el régimen de precipitaciones es básicamente estacional (por lo general entre octubre y marzo). Por consiguiente, la vegetación que caracteriza a estas regiones es la de tipo xerofita o sub xerofita.

Formaciones vegetales de la zona altoandina - El límite inferior de la zona altoandina se encuentra entre los 3800-4000 m. de altitud en el Centro y Sur de los Andes peruanos y de $3000 \mathrm{~m}$ en el Norte. Esta zona es pobre en plantas leñosas y estas se limitan (con excepción de unos cuantos arbustos) a determinados sitios, principalmente en superficies rocosas y pedregales. De acuerdo con el predominio de determinadas forma de vida y de crecimiento y a los tipos de suelo, se observan las siguientes formaciones vegetales (las orquídeas que se indican a continuación han sido registrados entre los $6^{\circ} 30^{\prime}$ y $9^{\circ} 30^{\prime} \mathrm{S}$ en la Cordillera Occidental de los Andes):

Pajonal - Ésta formación vegetal es la más extensa de la zona altoandina. Está compuesta por asociaciones de gramíneas que crecen en manojos o macollos de hojas duras y punzantes que corresponde a los géneros: Festuca, Stipa, Calamagrostis y Poa. Las orquídeas que se han registrado en los pajonales son: $A a$ aurantiaca, Aa erosa, Aa sp., Myrosmodes nubigenum, Myrosmodes paludosa, Myrosmodes sp., Pterichis triloba, y Pterichis sp.

Bofedal - Esta comunidad vegetal ocupa los lugares semipantanosos o pantanosos de la zona altoandina. En algunos bofedales la especie dominante es Distichia muscoides Nees y Meyen (Juncaceae), que crece a manera de cojines convexos; varias hierbas pequeñas mas no arbustos pueden acompañar a las Distichia. En ésta formación vegetal, sólo especies del género Myrosmodes han sido registrados; por ejemplo: Myrosmodes nubigenum, M. paludosa y M. pumilio.

Matorral - Son formaciones abiertas de arbustos con escasos arboles, helechos y hierbas anuales y geófitos. Las orquídeas registradas en esta formación son: $A a$ sp., Altensteinia fimbriata, Chloraea septentrionalis, Cranichis sp., Cyclopogon elatus, Cyrtochilum aureum, Elleanthus amethystinus, Elleanthus conifer, Epidendrum inamoenum, Epidendrum stictoglossum, Gomphichis macbridei, Gomphichis valida, Stenoptera montana, Stenoptera aff. peruviana, Masdevallia amabilis, Pleurothallis spiralis, Pleurothallis virgata, Pterichis triloba, Pterichis sp., Sudamerlycaste sp, Sauroglossum schweinfurthianum y Stelis sp.

En matorrales que se encuentran cerca a pequeños ríos y quebradas a altitudes superiores a $\operatorname{los} 3500$ m.; se ha registrado Liparis elegantula y Malaxis andicola creciendo bajo la sombra de algunos árboles y arbustos.

Vegetación de los pedregales y roquedales - Es la vegetación que se desarrolla en los acantilados, 
afloramientos rocosos y pedregales; está compuesta por líquenes, musgos, helechos, hierbas y algunos arbustos. Las orquídeas que habitan en estas formaciones crecen en la tierra que se acumula entre las piedras y en las fisuras de las rocas, y por lo general están protegidas por otras plantas. Las orquídeas registradas para ésta formación vegetal son: Aa sp. Chloraea septentrionalis, Cyrtochilum aureum, Gomphichis valida., Elleanthus amethystinus, Epidendrum stictoglossum, Masdevallia amabilis, Masdevallia semiteres, Platystele rauhii, Pleurothallis virgata, Pterichis triloba, Stenoptera montana, Stenoptera aff. peruviana, Stelis spp., Sudamerlycaste sp. y Trichoceros tupaipi.

También se han registrado orquídeas creciendo directamente sobre la roca (Fig. 1B), sin la protección de otras plantas, sólo líquenes y hay veces musgos están cubriendo sus raíces. Estas especies son: Masdevallia semiteres, Pleurothallis virgata, Stelis spp. y Trichoceros tupaipi.

Bosques -- De este tipo formación vegetal, la más importante es el bosque de Polylepis spp. Estas especies se desarrollan en ciertos valles altoandinos o laderas con suelos rocosos entre los 1800 y 5100 m (Mendoza \& Cano 2011). En los lugares donde los individuos de Polylepis no son muy densos se han registrado las siguientes orquídeas: Epidendrum stictoglossum, Gomphichis valida, Malaxis andicola, Masdevallia amabilis y Pleurothallis spiralis.

Por encima de los $3700 \mathrm{~m}$ de altitud, en las ramas de Polylepis weberbauer Pilg. y Buddleja incana Ruiz \& Pav. se ha registrado Epidendrum excelsum; ésta especie es la única orquídea epifita altoandina registrada hasta el momento.

En algunos paisajes antropizados, es decir lugares donde la vegetación ha sido alterada por la actividad humana; también se han registrado orquídeas (Tabla 2). Las zonas de los paisajes antropizados donde se han colectado orquídeas son: los bordes y cercos de los campos de cultivo, bordes de caminos y muros de las construcciones precolombinas abandonadas. Entre las orquídeas registradas en éstas zonas tenemos: Altensteinia fimbriata, Chloraea septentrionalis (Fig. 1C), Cyclopogon elatus, Cyrtochilum aureum, Stenoptera aff. peruviana, Masdevallia semiteres, Pleurothallis virgata y Stelis sp. (el total de especies registradas se muestra en la Tabla 2).
Formaciones vegetales de los valles interandinos Son los valles que se encuentra entre las estribaciones andinas entre los 900 y $3900 \mathrm{~m}$. de altitud; por donde corren los ríos que se dirigen hacia la cuenca del Amazonas. La cantidad de agua que reciben estos valles es muy variada; algunos gozan de humedad durante todo el año, mientras que otros soportan moderados periodos de sequía. A ésta última, corresponde la mayoría de los valles interandinos del Perú; por lo que el tipo de vegetación predominante es el matorral xerofítico.

Matorral xerofitico - Entre las especies leñosas que componen ésta formación vegetal se encuentran las pertenecientes a los géneros Acacia, Cercidium, Tecoma, Jatropha, Monnina, Rubus y Croton, y entre las herbáceas varias Cactáceas y Bromeliáceas.

Como ejemplo de las orquídeas de los matorrales xerofíticos de los valles interandinos citare a las especies registradas en el valle del Huallaga entre los 2000 y 3150 m de altitud; estas son: Aa sp., Altensteinia fimbriata, Cranichis ciliata, Epidendrum secundum, Malaxis andicola, Porphyrostachys pilifera (Fig. 1D), Pelexia aff. laxa, Ponthieva aff. pseudoracemosa, Sauroglossum schweinfurthianum y Trichoceros sp.

Muchos de los matorrales de los valles interandinos han sido impactados por la actividad del ser humano, se estima que gran parte de estas formaciones surgieron como resultado de la alteración de los bosques a causa de la agricultura, el pastoreo y la extracción de madera (Sarmiento 2002). La forestación o reforestación de los valles interandinos con especies exóticas; como Eucalyptus globulus Labill., provoca cambios en la composición de la flora nativa. Sin embargo, el grado del impacto varía de acuerdo a la zona donde ocurren estos bosques y a las dimensiones de los mismos (FAO 2002). En el sotobosques de plantaciones de E. globulus, en cuyas cercanías todavía existían matorrales con especies nativas, se han registrado las siguientes orquídeas: Cranichis ciliata, Malaxis andicola (Fig. 1E), Pelexia aff. laxa, Ponthieva aff. pseudoracemosa, Sauroglossum schweinfurthianum y Trichoceros sp.

Todavía existen muchas localidades de los Andes peruanos donde no se han realizado estudios florísticos; y si estos se han realizado, no han reportado especies de Orchidaceae. Posiblemente, esto se debe a que muchas veces la época cuando se hicieron los trabajos 
TABLA 2. Especies de orquídeas registradas para las diferentes formaciones vegetales de la zona altoandina y valle interandino. A.Alt $=$ Área Alterada, Bof $=$ Bofedal, B.Euc $=$ Bosque de Eucalyptus, B.Poly $=$ Bosque de Polylepis, Mat $=$ Matorral, MatXero $=$ Matorral xerofítico, Paj $=$ Pajonal, $P \& R=$ Pedregal y Roquedal.

\begin{tabular}{|c|c|c|c|c|c|c|c|c|}
\hline \multirow[b]{2}{*}{ Especies } & \multicolumn{6}{|c|}{ Zona altoandina } & \multicolumn{2}{|c|}{ Valle interandino } \\
\hline & Paj & Bof & Mat & P\&R & B.Poly & A.Alt & MatXero & B.Euc \\
\hline \multicolumn{9}{|l|}{ TRIBU CRANICHIDEAE } \\
\hline \multicolumn{9}{|l|}{ Subtribu Cranichidinae } \\
\hline Cranichis ciliata Kunth & & & & & & & $x$ & $x$ \\
\hline Cranichis sp. & & & $x$ & & & $x$ & & \\
\hline Ponthieva aff. pseudoracemosa Garay & & & & & & & $x$ & $x$ \\
\hline Pterichis triloba (Lindl.) Schltr. & $x$ & & $x$ & $x$ & & & & \\
\hline Pterichis sp. & $x$ & & $x$ & & & & & \\
\hline \multicolumn{9}{|l|}{ Subtribu Prescottinae } \\
\hline Aa aurantiaca D.Trujillo & $x$ & & & & & & & \\
\hline Aa erosa (Rchb.f.) Schltr. & $x$ & & & & & & & \\
\hline Aa spp. & $x$ & & $x$ & $x$ & & $\times$ & $x$ & \\
\hline Altensteinia fimbriata Kunth & & & $x$ & & & $x$ & $x$ & \\
\hline Gomphichis macbridei C. Schweinf. & & & $x$ & & & & & \\
\hline Gomphichis valida Rchb.f. & & & $x$ & $x$ & $x$ & $x$ & & \\
\hline Myrosmodes nubigenum Rchb.f. & $x$ & $x$ & & & & & & \\
\hline Myrosmodes paludosa (Rchb.f.) P.Ortiz & $x$ & $x$ & & & & & & \\
\hline Myrosmodes pumilio (Schltr.) C.A.Vargas & & $x$ & & & & & & \\
\hline Myrosmodes sp. & $\times$ & $\times$ & & & & & & \\
\hline Porphyrostachys pilifera (Kunth) Rchb.f. & & & & & & & $x$ & \\
\hline Stenoptera aff. peruviana C. Presl & & & $\times$ & $\times$ & & $\times$ & & \\
\hline Stenoptera montana C. Schweinf. & & & $x$ & $x$ & & $x$ & & \\
\hline \multicolumn{9}{|l|}{ Subtribu Spiranthinae } \\
\hline Cyclopogon elatus (Sw.) Schltr. & & & $x$ & & & $x$ & & \\
\hline Pelexia aff. laxa (Poepp. \& Endl.) Lindl. & & & & & & & $\times$ & $\times$ \\
\hline Sauroglossum schweinfurthianum Garay & & & $\times$ & & & $\times$ & $\times$ & $x$ \\
\hline \multicolumn{9}{|l|}{ TRIBU MALAXIDEAE } \\
\hline Liparis elegantula Kraenzl. & & & $x$ & & & & & \\
\hline Malaxis andicola (Ridl.) Kuntze & & & $x$ & & $x$ & & $x$ & $x$ \\
\hline \multicolumn{9}{|l|}{ TRIBU CHLORAEAE } \\
\hline Chloraea septentrionalis M.N. Correa & & & $x$ & $x$ & & $\times$ & & \\
\hline \multicolumn{9}{|l|}{ TRIBU SOBRALIEAE } \\
\hline Elleanthus amethystinus (Rchb.f. \& Warsz.) Rchb.f. & & & $x$ & $x$ & & $\times$ & & \\
\hline Elleanthus conifer (Rchb.f. \& Warsz.) Rchb.f. & & & $x$ & & & & & \\
\hline
\end{tabular}


TABLA 2. (continúa).

\begin{tabular}{|c|c|c|c|c|c|c|c|c|}
\hline \multirow[b]{2}{*}{ Especies } & \multicolumn{6}{|c|}{ Zona altoandina } & \multicolumn{2}{|c|}{ Valle interandino } \\
\hline & Paj & Bof & Mat & P\&R & B.Poly & A.Alt & MatXero & B.Euc \\
\hline \multicolumn{9}{|l|}{ TRIBU EPIDENDREAE } \\
\hline \multicolumn{9}{|l|}{ Subtribu Laeliinae } \\
\hline Epidendrum excelsum C.Schweinf. & & & & & $x$ & $x$ & & \\
\hline Epidendrum inamoenum Kraenzl. & & & $x$ & & & & & \\
\hline Epidendrum stictoglossum Hágsater \& D.Trujillo & & & $\times$ & $x$ & $\times$ & $x$ & & \\
\hline Epidendrum secundum Jacq. & & & & & & & $\times$ & \\
\hline \multicolumn{9}{|l|}{ Subtribu Pleurothallidinae } \\
\hline Masdevallia amabilis Rchb.f. \& Warsz. & & & $\times$ & $\times$ & $\times$ & & & \\
\hline Masdevallia semiteres Luer \& R.Escobar & & & & $x$ & & $x$ & & \\
\hline Platystele rauhii Luer & & & & $\times$ & & $\times$ & & \\
\hline Pleurothallis spiralis (Ruiz \& Pav.) Lindl. & & & $x$ & & $\times$ & & & \\
\hline Pleurothallis virgata Luer & & & $x$ & $x$ & & $x$ & & \\
\hline Stelis spp. & & & $\times$ & $\times$ & & $x$ & & \\
\hline \multicolumn{9}{|l|}{ TRIBU CYMBIDIEAE } \\
\hline \multicolumn{9}{|l|}{ Subtribu Maxillariinae } \\
\hline Sudamerlycaste sp. & & & $\times$ & $\times$ & & $x$ & & \\
\hline \multicolumn{9}{|l|}{ Subtribu Oncidiinae } \\
\hline Cyrtochilum aureum (Lindl.) Senghas & & & $\times$ & $\times$ & & $x$ & & \\
\hline Trichoceros tupaipi Rchb.f. & & & & $\times$ & & $x$ & & \\
\hline Trichoceros sp. & & & & & & & $x$ & $x$ \\
\hline
\end{tabular}

de campo no coincidieron con la época de floración de las orquídeas, o porque no se exploraron bien las formaciones vegetales dónde estas especies habitan. Algunas veces aunque se han observado especies de orquídeas en ciertas localidades, el material colectado no fue revisado por los especialistas de la familia y no se determinaron las especies; o simplemente no se prepararon especímenes de herbario. Todo esto ha dado como resultado, que exista poca información sobre la riqueza de especies y se subestime la diversidad de orquídeas del territorio andino y costero.

El primer paso para conservar las orquídeas de los Andes peruanos es conocer su diversidad y distribución; para ello es necesario muchos estudios de investigación básica que incluyan exploraciones botánicas en todas las formaciones vegetales; incluso en aquellas que han sido alteradas por el hombre.
Agradecimientos. A los curadores de los herbarios USM, MOL, HAO, HUT, CPUN y PRG por las facilidades brindadas durante la revisión de sus colecciones. A Hernando Layza Vera por su colaboración durante los trabajos de campo en el departamento de La Libertad y Cajamarca. A Asunción Cano y demás investigadores del Laboratorio de Florística de USM por colaborar en los últimos años con información y registros. A Oliver Whaley y Alfonso Orellana por compartir información sobre las lomas del departamento de Ica. A Carlos Vargas y Wilfredo Mendoza por las correcciones y mejoras en el texto.

\section{LiTERATURA CITADA}

Aguirre, Z., R. Linares-Palomino \& L.P. Kvist. 2006. Especies leñosas y formaciones vegetales en los bosques estacionalmente secos de Ecuador y Perú. Arnaldoa 13: 324-350. 
Cribb, P. 2003. Pterichis. Pp. 53-55 in: A.M. Pridgeon, P.J. Cribb, N.W. Chase, and F.N. Rasmussen, eds. Genera Orchidacearum, 3: Orchidoideae part 2, Vanilloideae. Oxford University Press.

Dillon, M.O., M. Nakazawa \& S. Leiva. 2003. The lomas formations of coastal Peru: composition and biogeographic history. Pp. 1-9 in: J. Haas \& M.O. Dillon (eds.), El Niño in Peru: biology and culture over 10,000 years. Fieldiana, Bot. 43.

FAO. 2002. Bibliografía anotada sobre los efectos ambientales, sociales y económicos de los Eucaliptos (versión en español), Documentos de Trabajo: Plantaciones forestales. Documento de Trabajo FP/17S. Palmberg-Lerche, C. (septiembre de 2002). Servicio de Desarrollo de Recursos Forestales, Dirección de Recursos Forestales, FAO, Roma.

Ferreyra, R. 1983. Los tipos de vegetación de la costa peruana. Anales Jard. Bot. Madrid 40: 241-256.

Ferreyra, R. 1993. Registros de la vegetación en la costa peruana en relación con el Fenómeno El Niño. Bull. Inst. fr. etudes andines 22: 259-266.

Hesse, R. 2012. Spatial distribution of and topographic controls on Tillandsia fog vegetation in coastal southern Peru: Remote sensing and modeling. J. Arid Environ. 78: 33-40.

La Torre-Cuadros, M. de los A. \& R. Linares-Palomino. 2008. Mapas y clasificación de vegetación en ecosistemas estacionales: un análisis cuantitativo de los bosques secos de Piura. Rev. Peru. Biol. 15: 31-42

Leiva, S., M. Zapata, G. Gayoso, P. Lezama, V. Quipuscoa \& M.O. Dillon. 2008. Diversidad florística de la Loma
Mongón, provincia de Casma, departamento Ancash, Perú. Arnaldoa 15: 45-62.

Linares-Palomino, R. 2004. Los bosques tropicales estacionalmente secos: II. Fitogeografía y composición florística. Arnaldoa 11: 103-138.

Mendoza, W. \& A. Cano. 2011. Diversidad del género Polylepis (Rosaceae, Sanguisorbeae) en los Andes peruanos. Rev. Peru. Biol. 18: 197- 200.

Pupulin, F. 2012. The Orchidaceae of Ruiz \& Pavón's "Flora Peruviana et Chilensis". A taxonomic study. I. Anales Jard. Bot. Madrid 69: 21-79.

Ruiz, H. 1952. Relación histórica el viaje, que hizo a los Reynos de Perú y Chile el botánico D. Hipólito Ruiz en el año de 1777 hasta el de 1788. Madrid. Edición y prologo Jaime J. Jaramillo. Real Academia de Ciencias Exactas, Físicas y Naturales, Madrid.

Sarmiento, F.O. 2002. Impulsores de cambio del paisaje: Dinámica de las líneas de árboles en la montología neotropical. Ecotropicos 15: 129-146.

Schweinfurth, C. 1958. Orchids of Peru. Fieldiana, Bot. 30: $1-260$.

Trujillo, D. \& A. Delgado. 2011. Aa from lomas formations. New Orchidaceae record from the desert coast of Peru. Lankesteriana 11: 33-38.

Vector-Peru SAC. 2009. Estudio de impacto ambiental del proyecto de explotación minera $\square$ Mina Justa $\square$ de Marcobre SAC. Resumen ejecutivo y plan de participación ciudadana.

Weberbauer, A. 1945. El mundo vegetal de los Andes peruanos. Estudio fitogeográfico. Ministerio de Agricultura, Lima. 